\title{
Minichromosome maintenance protein 7 as a potential prognostic factor for progression-free survival in high-grade serous carcinomas of the ovary
}

\author{
Takayo Ota ${ }^{1}$, Amy C Clayton ${ }^{1}$, Douglas M Minot ${ }^{1}$, Viji Shridhar ${ }^{1}$, Lynn C Hartmann ${ }^{2}$,
} C Blake Gilks ${ }^{3}$ and Jeremy R Chien ${ }^{1}$

${ }^{1}$ Department of Laboratory Medicine and Pathology, Mayo Clinic, Rochester, MN, USA; ${ }^{2}$ Division of Medical Oncology, Mayo Clinic, Rochester, MN, USA and ${ }^{3}$ Genetic Pathology Evaluation Centre of the Prostate Research Centre, Department of Pathology, Vancouver General Hospital and British Columbia Cancer Agency, Vancouver, BC, Canada

\begin{abstract}
Minichromosome maintenance protein 7 (MCM7) is involved in replicative licensing and synthesis of DNA. It was previously identified as an overexpressed gene in high-grade serous carcinomas compared with serous borderline tumors of the ovary in CDNA microarray studies. In this study, we sought to validate MCM7 expression in 342 ovarian tumors on tissue microarrays. MCM7 expression was quantified as the MCM7 labeling index, and it was independently generated by two methods: a score provided by manual review of each sample by a pathologist observer and by an automated cellular imaging system. Analyses of MCM7 scores indicated a high degree of concordance and distribution between the observer- and machine-generated MCM7 labeling indexes. MCM7 expression was significantly higher in high-grade serous carcinomas than in serous borderline tumors or other histological subtypes of ovarian cancer. For both observer- and machine-derived scores, univariate analyses indicated the significant association of a high MCM7 labeling index with better progressionfree survival in high-grade serous carcinomas. These results suggest the clinical importance of MCM7 expression in high-grade serous carcinomas of the ovary and the need for further evaluation of MCM7 as a potential prognostic factor in ovarian cancer.

Modern Pathology (2011) 24, 277-287; doi:10.1038/modpathol.2010.202; published online 12 November 2010
\end{abstract}

Keywords: immunohistochemistry; MCM7; ovarian cancer

Ovarian cancer is the leading cause of death among gynecologic malignancies in North America. ${ }^{1}$ In 2009, 21550 new cases of ovarian cancer were expected in the United States. The prognosis remains poor, with a 5 -year overall survival rate of $35 \%$. Tumor stages, residual diseases after surgery, histological types, and tumor grades are the most important prognostic factors (reviewed by Gadducci et $a l^{2}$ ). In addition to these predictors, cDNA microarray analyses have identified several cell

Correspondence: Dr JR Chien, PhD, Department of Laboratory Medicine and Pathology, Mayo Clinic, 200 First Street SW, Rochester, MN 55905, USA.

E-mail: chien.jeremy@mayo.edu

Received 9 June 2010; revised 12 August 2010; accepted 12 August 2010; published online 12 November 2010 cycle-related proteins as potential biomarkers to predict response to chemotherapy and prognostic clinical outcomes. ${ }^{3,4}$

cDNA microarray analyses have been used as high-throughput screening tools to explore gene expression, which can be used as a gene signature to detect ovarian cancer at an early stage, predict clinical outcomes, and identify additional therapeutic targets. ${ }^{5-8}$ In our previous study, we compared gene expression profiles between stage I serous borderline tumors and high-grade stage I serous cancer, and we identified the minichromosome maintenance protein 7 (MCM7) as an upregulated transcript in serous carcinomas compared with serous borderline tumors. ${ }^{8}$

MCM proteins are highly conserved proteins from yeast to eukaryotic organisms, which have an 
important role in DNA synthesis (reviewed by Tye $^{9}$ and Forsburg ${ }^{10,11}$ ). MCM proteins are involved in two critical steps in DNA synthesis: the first step is DNA replication initiation mediated by hexametric MCM complexes at replication origins, and the second step is DNA elongation mediated by MCM helicase activities. MCM7 can be used as a prognostic indicator in various cancers, such as endometrial cancer, ${ }^{12}$ prostate cancer,${ }^{13}$ neuroblastoma, ${ }^{14}$ colorectal cancer, ${ }^{15}$ and small cell lung cancer. ${ }^{16}$ In endometrial, prostate, colon, and lung cancer, a high MCM7 labeling index has a worse prognosis in cumulative overall survival. In neuroblastoma, ${ }^{14}$ high MCM7 expression without MYCN amplification shows a better prognosis for progression-free survival. In glioblastoma, MCM7 expression is not correlated with its clinical outcome. ${ }^{17}$ These differences might be attributable to the differences in cancer characteristics, end points, and sample sizes in these studies. Moreover, the MCM7 staining pattern is related to the thickness and invasiveness of malignant melanocytic skin lesions. ${ }^{18}$ The thickness and invasiveness of the disease in melanoma determine the prognosis of the disease; thus, MCM7 is a useful marker for predicting disease prognosis.

In this study, we investigated MCM7 expression in epithelial ovarian carcinomas and correlated its expression with pathologic factors using two different MCM7 labeling indexes produced by a pathologist observer and by the automated cellular imaging system on tumor microarrays from 342 patients.

\section{Materials and methods}

\section{Tissue Microarrays}

Tissue microarrays were constructed from duplicate 0.6-mm cores, using cases harvested, with patient consent, for the Gynecologic Tissue Bank at Vancouver General Hospital. Ethical approval was obtained from the University of British Columbia. Tumor tissue samples, all collected since 2003, were promptly fixed in $10 \%$ neutral buffered formalin and embedded in formalin after tissue processing. The tissue microarrays were constructed with a microarrayer (Beecher Instruments, Silver Springs, MD, USA). Patients with ovarian carcinoma were treated according to provincial treatment guidelines, which include platinum/taxane chemotherapy for all patients except those with stage IA and IB tumors, who do not routinely receive chemotherapy. Histopathology and tumor grade were reviewed and assigned according to the World Health Organization (WHO) and Silverberg criteria, respectively. Clear-cell carcinomas were all considered grade 3 , as recommended by WHO. The patient age at surgery ranged from 22 to 100 years; the mean was 59.5 years. Follow-up ranged from 0.02 to 8.8 years; the mean was 2.9 years. The patient with 0.02 year of follow-up had serous borderline tumor, and she died perioperatively 1 week after the surgery. This record was censored in both overall survival and progression-free survival analyses.

\section{Immunohistochemical Analysis}

Sections of tissue arrays were deparaffinized, rehydrated, and submitted to antigen retrieval by a steamer for $25 \mathrm{~min}$ in target retrieval solution (Dako, Carpinteria, CA, USA). Endogenous peroxide was diminished with $3 \% \mathrm{H}_{2} \mathrm{O}_{2}$ for 30 min. Slides were blocked in protein block solution for $30 \mathrm{~min}$ and then blocked with avidin and biotin for $10 \mathrm{~min}$ each, followed by incubation with MCM7 antibody (ab2360; Abcam, Cambridge, MA, USA) 1:200 overnight at $4{ }^{\circ} \mathrm{C}$. The sections were then incubated with biotinylated universal link for $15 \mathrm{~min}$ and streptavidin for $25 \mathrm{~min}$ at $25^{\circ} \mathrm{C}$. Slides were developed in diaminobenzine and counterstained with hematoxylin.

\section{Pathologist Observer Review}

MCM7 staining in tumor samples was microscopically examined under $\times 10$ to $\times 20$ objective lenses, and immunopositive nuclei were estimated as the percentage of tumor nuclei, varying from 0 to $100 \%$ with incremental $5 \%$. All slides were analyzed in a blinded fashion.

\section{Automated Cellular Imaging System (ACIS)}

ACIS III is an image analysis system that analyzes immunohistochemically stained tissue using an automated bright-field microscope and proprietary analysis software. ACIS III (Dako) transforms an immunohistochemically stained slide into a digital image that, through various algorithms, can be used to detect, enumerate, and classify stained cellular objects. For this study, a tissue microarray application was used that allows for analysis of multiple tissue samples on the same slide. For homogenous tumor samples, the entire tissue core was analyzed by one observer and the percentage of positively stained nuclei was recorded. Operator-selected regions were collected from samples that contained abundant nontumor elements (ie, stroma, hematopoietic cells) by one observer (DMM). In all cases in which operator-selected regions were used, two areas were chosen that represented a pure tumor population and were collected using a $40 \times$ circle tool.

\section{Statistical Analyses}

The JMP program (version 8.0; SAS Institute, Cary, NC, USA) was used to perform all statistical analyses. Concordance between MCM7 labeling index produced by the observer and the ACIS was estimated using four different correlation analyses. 
MCM7 expression among groups was determined by pairwise comparison and the Student's $t$-test. The Cox proportional hazards model was used to assess the association of MCM7 expression and other clinical and pathological factors with disease progression and survival. Multivariate analysis was then performed to identify the independent prognostic factors as well as to estimate their effects on progression-free survival and overall survival adjusted for covariates (age, histology, and stage). Kaplan-Meier survival curves by MCM7 characteristics were calculated and compared using the logrank test. All statistical tests were two tailed, with a significance level set at $5 \%$.

\section{Results}

\section{Distribution of Tumor Samples by Stage and Histology}

To determine sample distribution by stage and histology, we generated a contingency table and plotted the distribution by histology color-coded stage (Figure 1). The majority of tumor samples in the tissue microarrays were advanced-stage, highgrade serous carcinomas. In contrast, a majority of tumors in the other histological subtypes (endometrioid, clear cell, and mucinous) presented at early stages (Figure 1). These data are consistent with the results from other studies using SEER data. ${ }^{19}$ Table 1

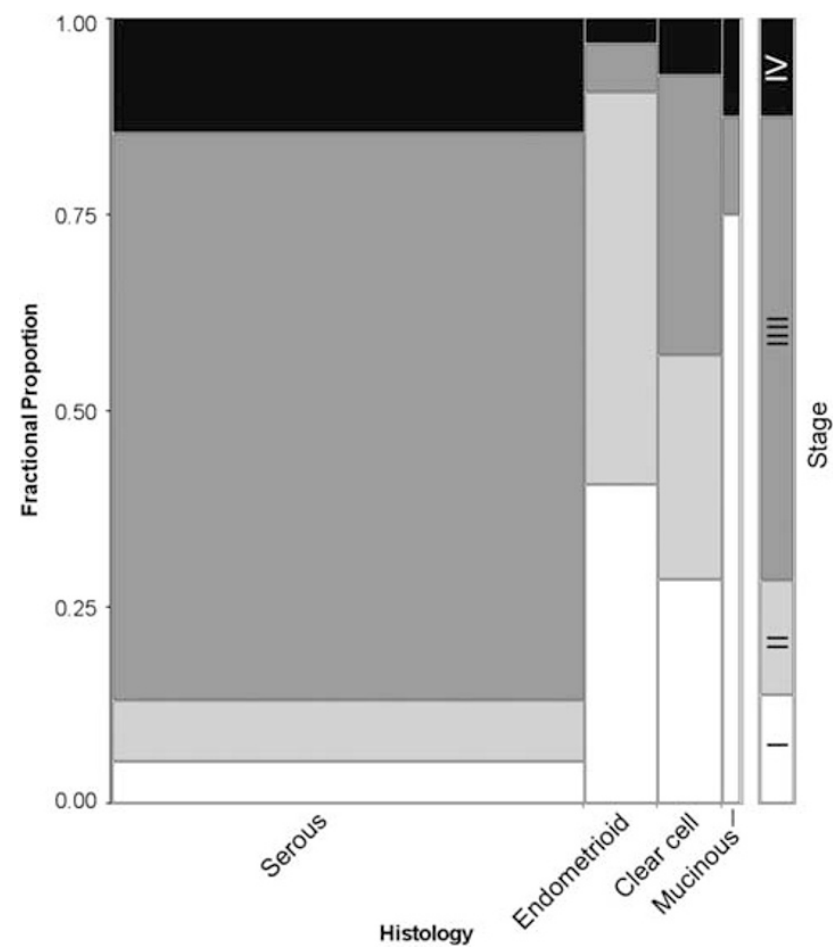

Figure 1 Distribution of tumor samples on the basis of histology (on $x$ axis) and stage (on $y$ axis). The width of each column represents the sample size. High-grade serous tumors contain the largest sample size, and mucinous tumors contain the smallest sample size. In addition, high-grade serous tumors had the highest percentage of patients with advanced disease.
Table 1 Histological distribution of specimens in tissue microarrays

\begin{tabular}{lrr}
\hline Histological diagnosis & No. & Percentage \\
\hline High-grade serous & 218 & 63.74 \\
Endometrioid & 34 & 9.94 \\
Clear cell & 29 & 8.48 \\
Mucinous & 10 & 2.92 \\
Undifferentiated & 9 & 2.63 \\
Low-grade serous & 9 & 2.63 \\
Serous borderline & 17 & 4.97 \\
Fallopian tube & 3 & 0.88 \\
Adenocarcinoma NOS & 13 & 3.80 \\
Total & 342 & 100.00 \\
\hline
\end{tabular}

NOS, not otherwise specified.

includes a detailed description and distribution of the tumors.

Variable Expression of MCM7 in the Tumor Nuclei of Tumor Samples

To analyze MCM7 expression in ovarian tumors, we performed immunohistochemical analysis using commercially available MCM7 antibodies. To set up the conditions for immunohistochemical analysis, we used mouse testicular tissue. As shown in Figure 2a, germ cells with proliferative capacity showed intense nuclear staining for MCM7, whereas mature sperm cells that lost proliferative capacity showed no staining for MCM7. These results demonstrated the specificity of MCM7 antibody. Therefore, similar conditions were used to perform immunohistochemical analysis of MCM7 on tissue microarrays. Immunohistochemical analysis of MCM7 expression indicated variable expression of MCM7 among tumor samples. MCM7 staining was restricted to nuclei, and nuclear expression of MCM7 was more prominent in tumor nuclei than in surrounding normal tissues (Figure 2b). Tumor nuclei that were positive for MCM7 staining were expressed as the percentage of total tumor nuclei. Representative MCM7 staining and the corresponding labeling indexes are shown in Figure $2 b$.

\section{Analysis of the MCM7 Labeling Index Showed Similar Distribution between Data Obtained by Human Observers and ACIS}

Quantile plots indicate quantized distribution of the MCM7 labeling index by the observer, but continuous distribution of the MCM7 labeling index by the ACIS (Figure 3). These differences reflect the systematic bias of human observers to produce the labeling index as multiples of five. Distribution of the binned MCM7 labeling index shows similar patterns of distribution between data produced by the observers and ACIS. Finally, a correlation analysis of MCM7 labeling indexes indicates a 
strong correlation between the two scoring modes (Figure 3 and Table 2).

\section{MCM7 Expression Is Higher in High-Grade Serous Carcinomas Than in Serous Borderline Tumors}

To validate our previous cDNA microarray studies indicating that MCM7 expression was higher in high-grade serous carcinomas than in serous borderline tumors, we analyzed MCM7 expression in highgrade and borderline tumor samples. The results, shown in Figure 4, indicate higher levels of MCM7 expression in high-grade serous carcinoma (histology code 1) compared with serous borderline tumors (histology code 7). Similar results were obtained using the ACIS score, which indicates that the finding was observer independent (Figure 4).

\section{MCM7 Expression Is Higher in High-Grade Serous Carcinomas Than in Other Histological Subtypes}

To determine MCM7 expression across various histological subtypes of ovarian tumors, we subgrouped MCM7 expression on the basis of histotypes and analyzed the variance among groups. The results, shown in Figure 5 and Table 3, indicate that MCM7 expression was significantly higher in highgrade serous carcinomas than in all other subtypes. In particular, significant differences in MCM7 expression were observed when high-grade serous and clear cells or high-grade serous and mucinous or high-grade serous tumors were compared with various other subtypes. Similar findings were observed when the ACIS score was obtained, suggesting that the results were observer independent.

\section{Higher MCM7 Expression Is Significantly Associated with Better Progression-Free Survival}

To determine the clinical importance of MCM7 expression, we performed Cox proportional hazards analysis to estimate the association between MCM7 expression and overall survival and progression-free survival. Likelihood ratio tests were performed to determine the significance of MCM7 expression in overall survival and progression-free survival outcomes. These analyses indicate that there was no significant association between MCM7 expression and overall survival, although disease stage was strongly associated with overall survival (Table 4). However, in multivariate analysis, stage and MCM7 labeling index were significantly associated with progression-free survival for the entire tumor cohort. Because serous histology represents the majority of the cohort, we determined whether MCM7 expression in high-grade serous tumors is associated with progression-free survival. These analyses indicate that the MCM7 labeling index was significantly associated with progression-free survival in patients with high-grade serous carcinomas (Table 4). In addition, in the multivariate model, the MCM7 labeling index produced by ACIS was independently associated with progression-free survival, whereas no significant association was observed with the MCM7 labeling index produced by observer review (Table 4). Further analysis of the multivariate model that included the MCM7 labeling index and two-stage classification (early and late stages) also yielded a significant association of ACIS-produced MCM7 labeling index with progression-free survival in late-stage, high-grade serous carcinomas (Table 4). These results indicate that the ACIS-produced MCM7 labeling index is an
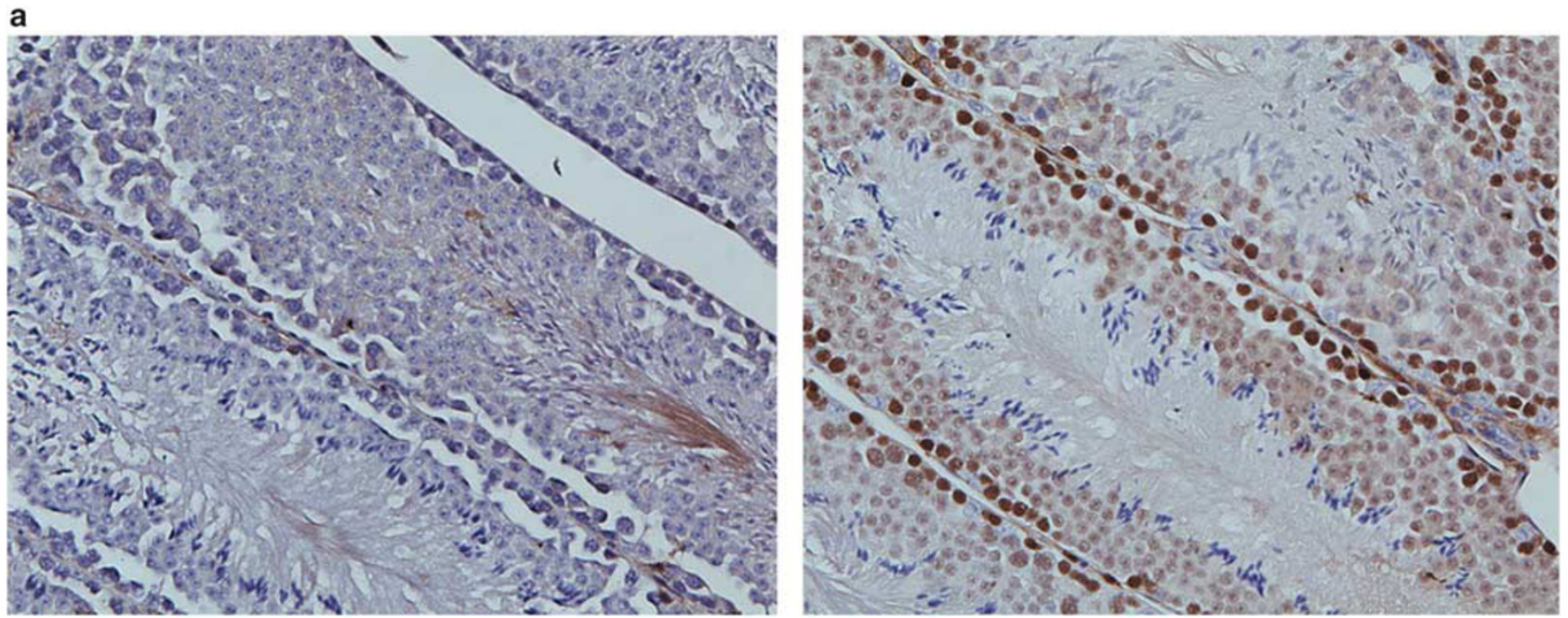

Figure 2 Immunohistochemical analysis of minichromosome maintenance protein 7 (MCM7) expression in tissue samples. (a) As positive and negative controls, mouse testicular specimens were stained with anti-MCM7 antibodies. MCM7 is specifically expressed in the nuclei of spermatogonia and spermatocytes. As the cells in the germinal layer differentiated, less staining of MCM7 was observed in spermatids, and fully differentiated sperms completely lacked MCM7 expression. (b) Representative nuclear staining patterns of MCM7 in tumor cells. MCM7 staining is observed by brown chromogen staining. All micrographs were taken at original magnification of $\times 10$ objective. 
b

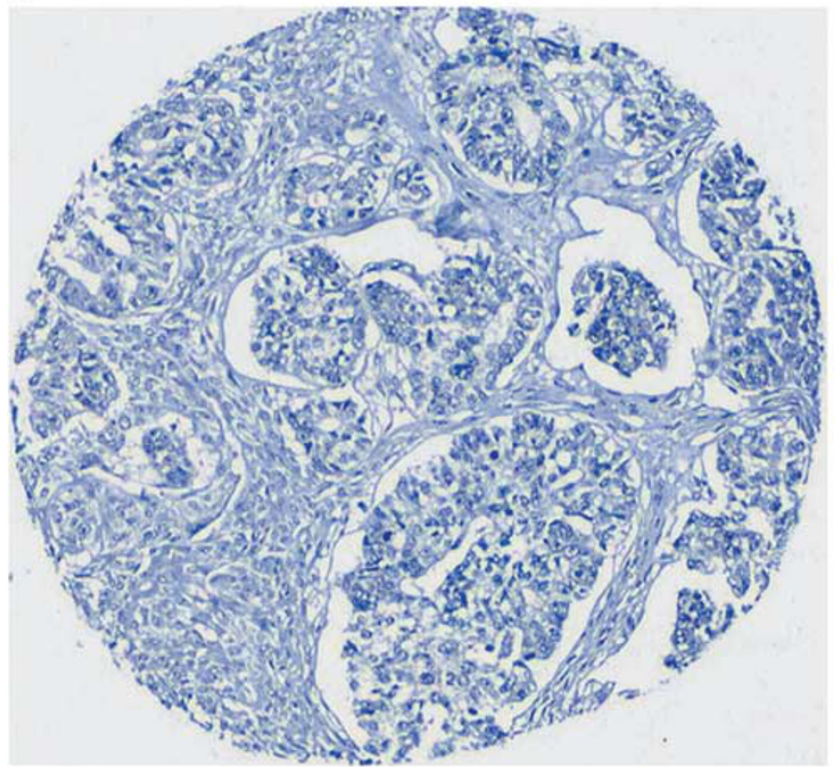

MCM7 labeling index $=0.39$

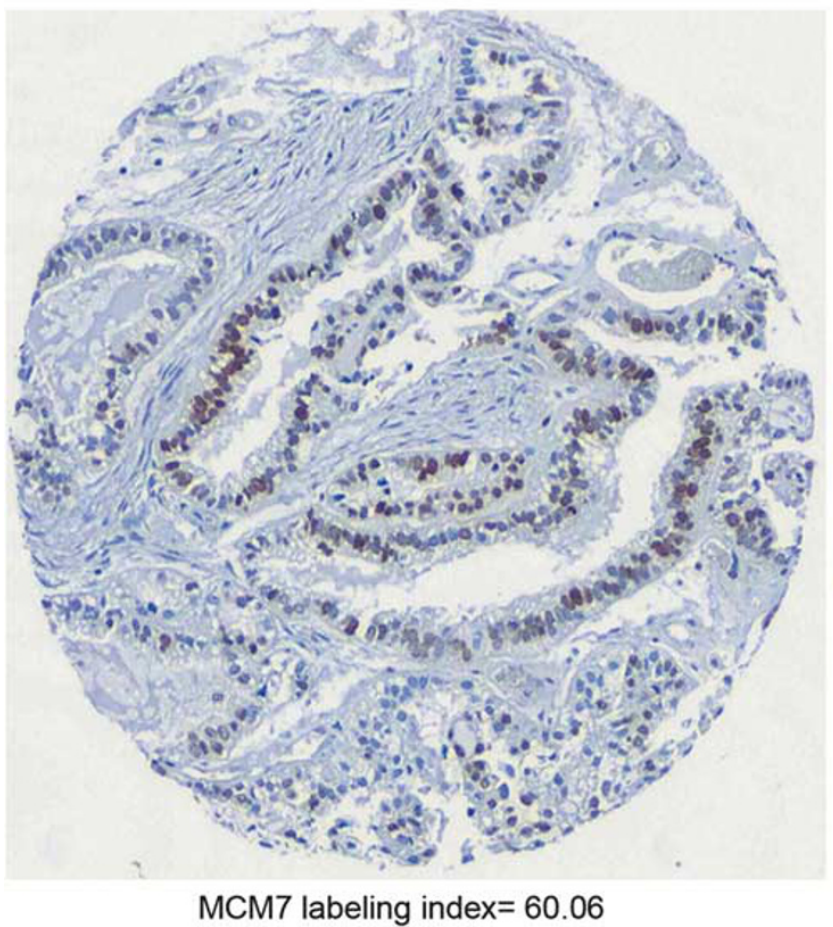

Figure 2 continued.

independent prognostic factor in late-stage, highgrade serous carcinomas of the ovary. We further dichotomized the MCM7 labeling index into two groups (high and low) on the basis of unbiased median distribution of the MCM7 labeling index for the entire tumor cohort. The MCM7 labeling index below the median score was considered low, and the MCM7 labeling index equal to or above the median score was considered high. The Kaplan-Meier progression-free survival analysis indicate that high

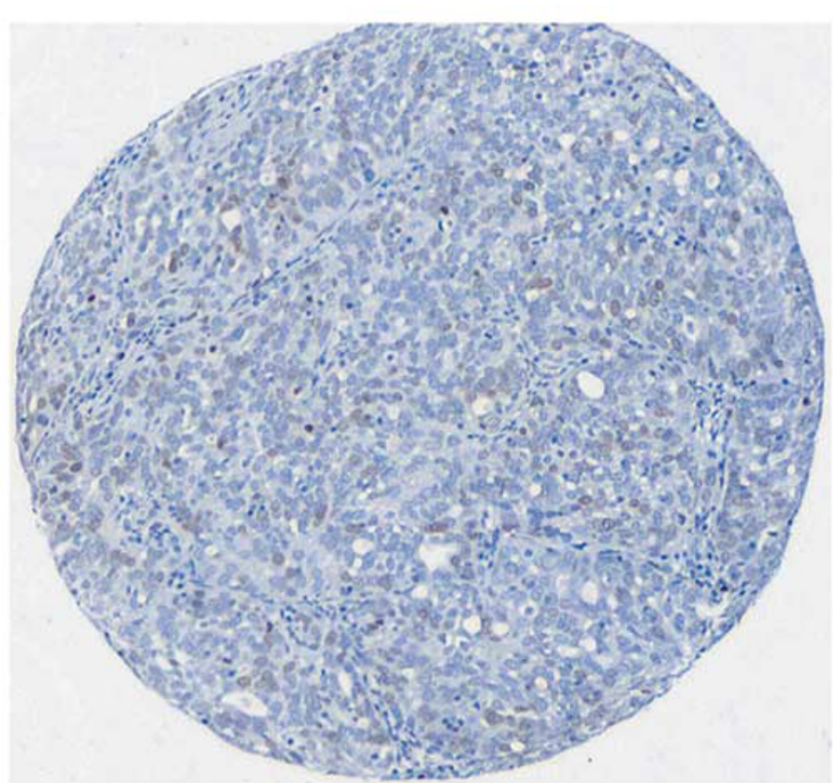

MCM7 labeling index $=27.18$

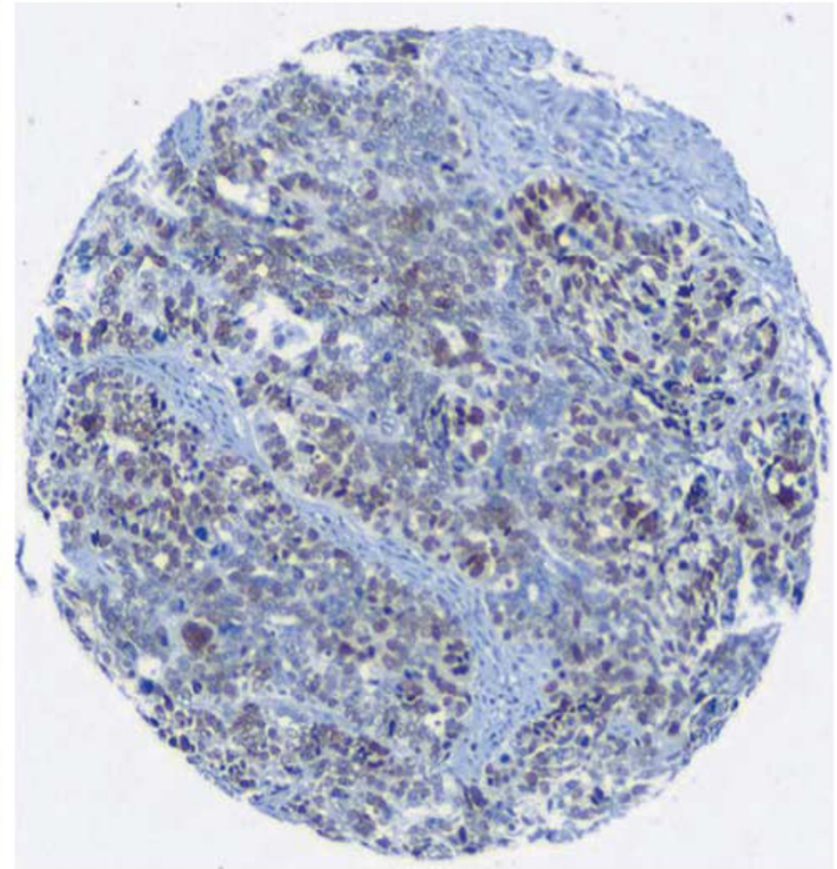

MCM7 labeling index $=85.50$

MCM7 labeling indexes were significantly associated with better progression-free survival in both methods of MCM7 scoring for the entire tumor cohort consisting of various histologies and stages (Figure 6). Again, because high-grade serous carcinomas represented the majority, we then limited the Kaplan-Meier progression-free survival analysis to high-grade serous carcinomas. The results from this analysis indicate that higher MCM labeling indexes were significantly associated with better 

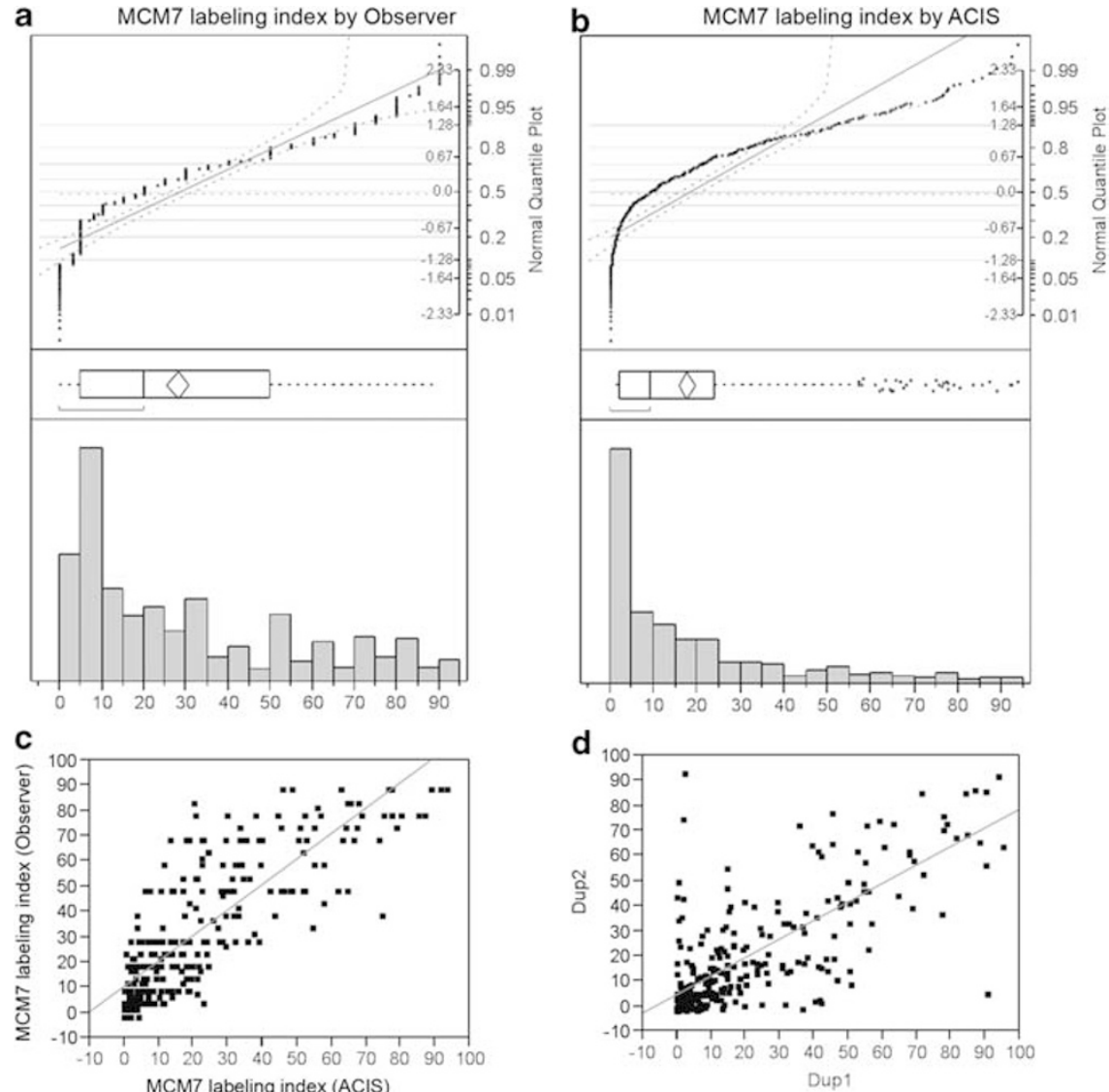

Figure 3 Distribution of minichromosome maintenance protein 7 (MCM7) labeling index by a pathologist observer (observer) and an automated cellular imaging system (ACIS). (a) Normal quantile plots and frequency distribution of binned data indicate skewed distribution of the MCM7 labeling index toward the lower end. Patterns of distribution are similar in both observer- and ACIS-derived data. (b) Correlative analysis between observer- and ACIS-derived MCM7 labeling indexes shows significant correlation. (c) Correlation between MCM7 labeling indexes produced by the observer and the ACIS. (d) Correlation between ACIS-derived MCM7 labeling indexes of duplicate samples from the arrays.

progression-free survival (Figure 6). Further analysis indicates that a significant association between MCM7 expression and progression-free survival was observed in late-stage (stage III and IV) serous carcinomas of the ovary (Table 4). Finally, risk ratio analyses indicated reduced risks of recurrence for patients with higher levels of MCM7 expression (Tables 5 and 6). In contrast, higher stages were associated with increased risk of recurrence. Interestingly, much reduced risk of recurrence was observed in patients with endometrioid carcinomas compared with patients with high-grade serous carcinomas, whereas slightly increased risk of recurrence was observed in patients with clear-cell carcinomas compared with patients with high-grade serous carcinomas (Table 7).

\section{Discussion}

In this study, we identified MCM7 to be overexpressed in high-grade serous carcinomas compared with serous borderline tumors or clear- cell carcinomas. Several reported mechanisms regulate MCM7 expression. The promoter regions of MCM5, MCM6, and MCM7 each contain numerous E2F activation sites, implying that E2F controls the Mcm levels at the G1/S boundary. ${ }^{20-22}$ In a mouse model for cervical cancer, MCM7 expression correlated with $\mathrm{Rb}$ inactivation, which bound to $\mathrm{E} 2 \mathrm{~F}$ and prevented it from stimulating cellular proliferation. In our previous cDNA microarray studies, $^{8}$ we identified MCM7 as an overexpressed transcript in carcinomas compared with serous borderline tumors. In the same study, we identified the E2F pathway to be a candidate biological pathway altered in high-grade serous carcinomas. Taken together, these results suggest that alterations in the E2F pathway may regulate MCM7 expression. In addition, the MCM7 promoter has an E-box binding site for the MYC oncoproteins ${ }^{20}$ MCM7 is a direct target of MYCN, and overexpression of MYCN increases MCM7 expression in neuroblastoma cell lines. ${ }^{23}$ Therefore, multiple biological pathways may be responsible for overexpression of MCM7 in highgrade serous carcinomas of the ovary. 
Table 2 Concordance between observer and ACIS MCM7 scores

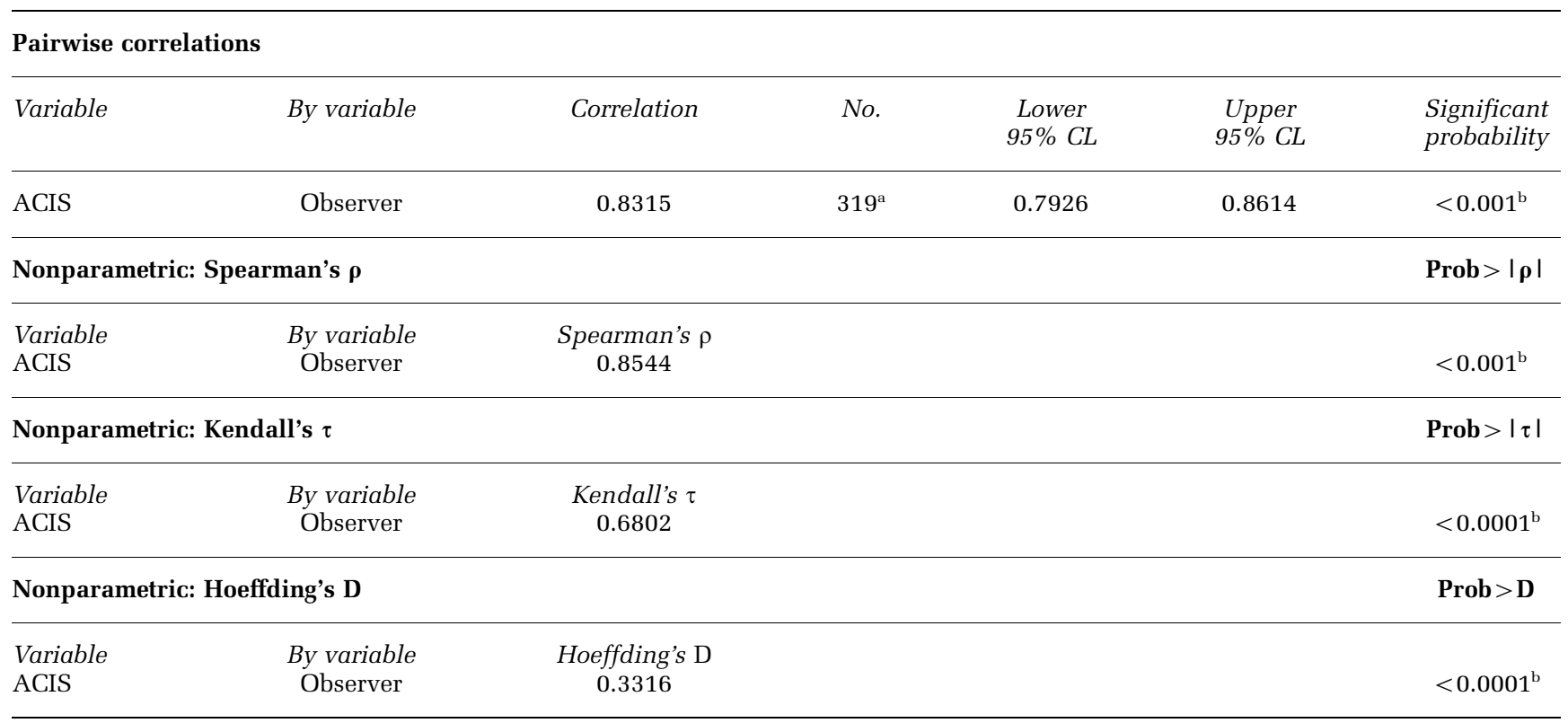

ACIS, automated cellular imaging system; CL, confidence limit; MCM7, minichromosome maintenance protein 7; Observer, pathologist observer; Prob, probability.

${ }^{\mathrm{a} O u t}$ of 342 samples, 319 have both observer and ACIS scores.

${ }^{\mathrm{b}}$ Significance at $\alpha<0.05$.
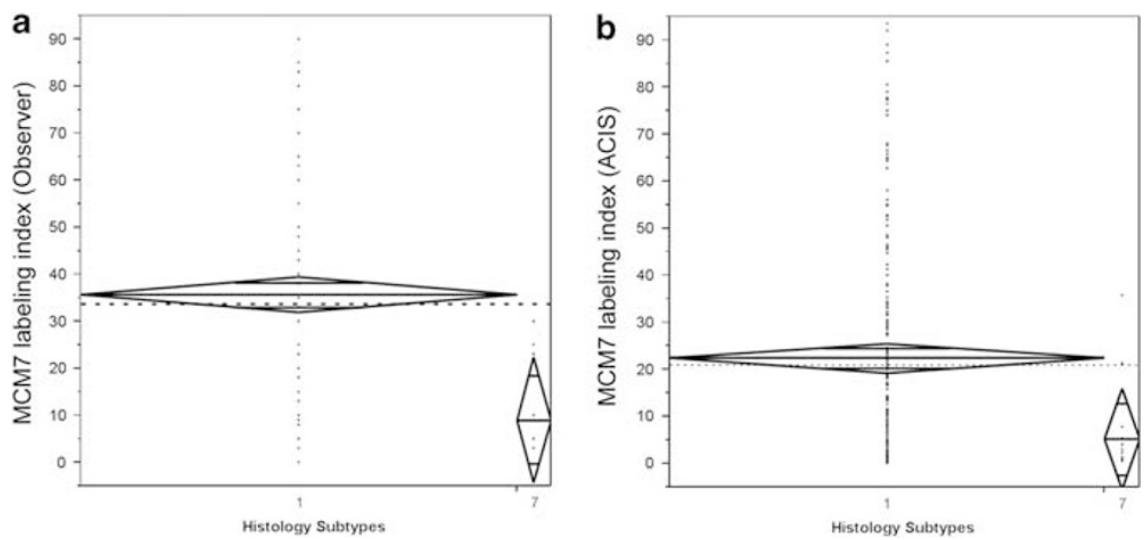

Figure 4 Minichromosome maintenance protein 7 (MCM7) expression is significantly higher in high-grade serous tumors (histology code 1, $n=218$ ) compared with serous borderline tumors (histology code $7, n=17$ ). Both observer- (a) and ACIS-derived (b) scores produced statistically significant differences $(P<0.001$ and $P=0.001)$ between the two groups. Results are presented as mean diamond comparison charts in which the group mean is represented by a horizontal line across the center of diamonds, the upper and lower $95 \%$ confidence limits are indicated by the tips of diamonds, and the overlap marks are indicated by two short horizontal lines flanking the mean.

Mcm expression represents cell cycle entry and is used as a proliferative marker superior to commonly used other proliferative markers, such as Ki67 and proliferating cell nuclear antigens (PCNAs), ${ }^{12,15-17}$ except melanoma. ${ }^{18}$ MCM7 is expressed in more cells than Ki67 or PCNAs, because it is expressed in cells licensed to proliferate in addition to those that are already proliferating. ${ }^{24}$ As chemotherapy is most effective against proliferating cells, ${ }^{25}$ it is conceivable that MCM7 expression may indicate responsiveness to chemotherapy that may in turn affect progression-free survival.
Our analysis of MCM7 expression in 342 tumor specimens on tissue microarrays indicates the prognostic potential of MCM7 expression in ovarian cancer, considering that its expression is significantly associated with progression-free survival, although no significant association was observed with overall survival. It should be noted that all patients in this cohort received a uniform standard of care, including optimal debulking surgery and platinum-based chemotherapy. Although several factors, including age, performance status, residual disease, and histology, are considered independent 

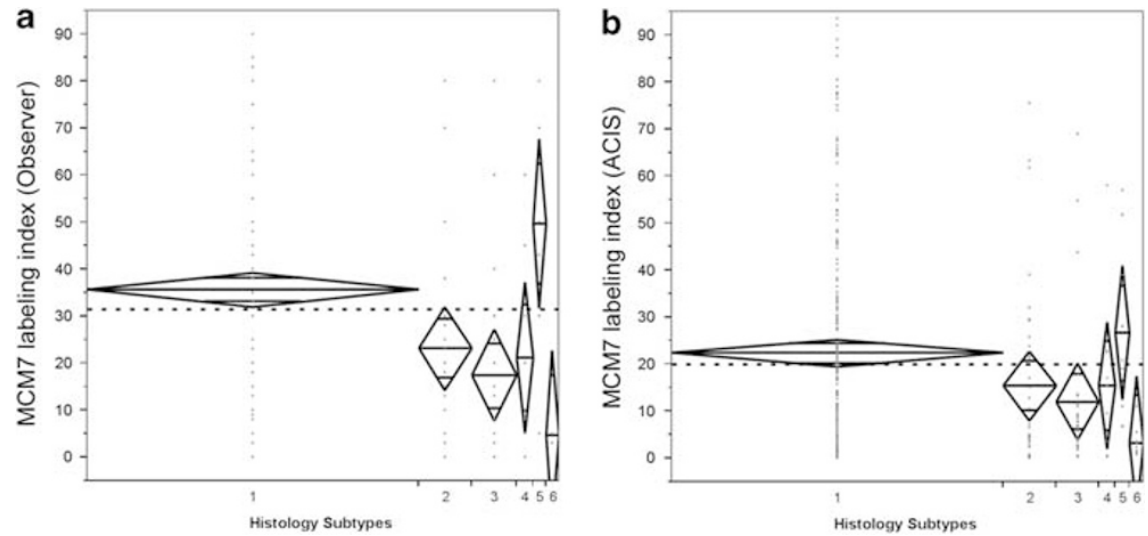

Figure 5 Highest expression of minichromosome maintenance protein 7 is detected in the most aggressive ovarian cancer subtype: highgrade serous carcinomas (histology code 1) compared with other subtypes. The numbers on the vertical axes represent MCM7 labeling index produced by the observer (a) and the ACIS (b). Results are presented as mean diamond comparison charts in which the group mean is represented by a horizontal line across the center of diamonds, the upper and lower $95 \%$ confidence limits are indicated by the tips of diamonds, and the overlap marks are indicated by two short horizontal lines flanking the means. Histology codes are as follow: $1=$ highgrade serous; $2=$ endometrioid; $3=$ clear cell; $4=$ mucinous; $5=$ undifferentiated; and $6=$ low-grade serous.

Table 3 Mean distribution of MCM7 labeling index by observer and ACIS among six histological subtypes

\begin{tabular}{|c|c|c|c|c|c|c|}
\hline & $\begin{array}{l}\text { Serous } \\
\text { (1) }\end{array}$ & $\begin{array}{c}\text { Endometrioid } \\
\text { (2) }\end{array}$ & $\begin{array}{l}\text { Clear cell } \\
\text { (3) }\end{array}$ & $\begin{array}{l}\text { Mucinous } \\
\quad \text { (4) }\end{array}$ & $\begin{array}{c}\text { Undifferentiated } \\
\text { (5) }\end{array}$ & $\begin{array}{l}\text { Low-grade } \\
\text { serous (6) }\end{array}$ \\
\hline \multicolumn{7}{|l|}{ Observer } \\
\hline Mean & 35.66 & 23.15 & 17.41 & 21.30 & 49.75 & 4.75 \\
\hline s.e.m. & 1.96 & 3.63 & 3.49 & 6.24 & 9.1 & 9.1 \\
\hline Upper 95\% mean & 39.53 & 30.55 & 24.58 & 35.41 & 67.66 & 22.66 \\
\hline Lower 95\% mean & 31.79 & 15.76 & 10.24 & 7.19 & 31.85 & -13.15 \\
\hline No. & 202 & 33 & 27 & 10 & 8 & 8 \\
\hline $\begin{array}{l}1 \text { vs } 2 \\
1 \text { vs } 3 \\
1 \text { vs } 6\end{array}$ & $\begin{array}{c}\text { P-value } \\
\mathbf{0 . 0 1} \\
<\mathbf{0 . 0 0 1} \\
\mathbf{0 . 0 0 1}\end{array}$ & & & & & \\
\hline \multicolumn{7}{|l|}{$A C I S$} \\
\hline Mean & 22.40 & 15.45 & 12.10 & 15.49 & 26.6645 & 3.3679 \\
\hline s.e.m. & 1.61 & 3.38 & 3.21 & 5.43 & 7.2381 & 7.2381 \\
\hline Upper 95\% mean & 25.56 & 22.33 & 18.71 & 27.77 & 40.91 & 17.613 \\
\hline Lower 95\% mean & 19.23 & 8.58 & 5.50 & 3.21 & 12.42 & -10.88 \\
\hline No. & 209 & 34 & 27 & 10 & 9 & 9 \\
\hline & $\mathrm{P}$-value & & & & & \\
\hline 1 vs 3 & 0.02 & & & & & \\
\hline 1 vs 6 & 0.01 & & & & & \\
\hline
\end{tabular}

ACIS, automated cellular imaging system; MCM7, minichromosome maintenance protein 7; Observer, pathologist observer.

${ }^{a}$ Each pair was analyzed using Student's $t$-test. Bold values represent statistically significant differences.

prognostic factors for progression-free survival, our findings indicate that the MCM7 labeling index is also a prognostic factor for progression-free survival, independent of age, stage, and histology. As performance status and residual disease volume were not available, we could not adjust for these independent prognostic factors.

Analysis of stage and histology distribution of the tumor cohort indicates appropriate representation of ovarian cancer cases in the US and Canadian population. For example, as expected, patients with high-grade serous carcinomas represented the majority. Moreover, the overwhelming majority of highgrade serous carcinomas were diagnosed at an advanced stage compared with other histology subtypes. These results are important because they indicate that our study is well represented and that the study outcomes may also reflect the general ovarian cancer patient population in the United States and Canada.

In general, we observed specific staining of MCM7 in the tumor nuclei. Analysis of the MCM7 labeling 
Table 4 Univariate and multivariate analysis of MCM7 labeling index

\begin{tabular}{|c|c|c|c|c|c|}
\hline & $\mathrm{P}$-value; observer & No. of events & P-value; ACIS & No. of events & Analysis \\
\hline \multicolumn{6}{|l|}{ Overall survival } \\
\hline All histology & & 81 & & 82 & Multivariate \\
\hline Stage & $<\mathbf{0 . 0 0 0 1}$ & & $<\mathbf{0 . 0 0 0 1}$ & & \\
\hline Histology & 0.093 & & 0.084 & & \\
\hline MCM7 & 0.71 & & 0.051 & & \\
\hline \multicolumn{6}{|l|}{ Serous } \\
\hline MCM7 & 0.77 & 74 & 0.97 & 75 & Univariate \\
\hline \multicolumn{6}{|l|}{ Progression-free survival } \\
\hline All histology & & 138 & & 141 & Multivariate \\
\hline Stage & $<0.0001$ & & $<\mathbf{0 . 0 0 0 1}$ & & \\
\hline Histology & 0.08 & & 0.1 & & \\
\hline MCM7 & 0.08 & & 0.015 & & \\
\hline Serous & 0.069 & 149 & 0.025 & 153 & Univariate \\
\hline 4-stage & $<0.0001$ & 142 & $<\mathbf{0 . 0 0 1}$ & 146 & Multivariate \\
\hline MCM7 & 0.11 & & 0.035 & & \\
\hline 2-stage & $<\mathbf{0 . 0 0 0 1}$ & & $<\mathbf{0 . 0 0 0 1}$ & & Multivariate \\
\hline MCM7 & 0.094 & & 0.033 & & \\
\hline Serous (low vs high) & & & & & Univariate \\
\hline Early & 0.29 & 17 & 0.26 & 18 & \\
\hline Late & 0.037 & 125 & 0.032 & 128 & \\
\hline
\end{tabular}

ACIS, automated cellular imaging system; MCM7, minichromosome maintenance protein 7; Observer, pathologist observer; 4-stage, FIGO 4-stage classification; 2-stage, early and late stage.

${ }^{\mathrm{a}}$ Log-rank analysis.

Significant associations are highlighted by bold letters.
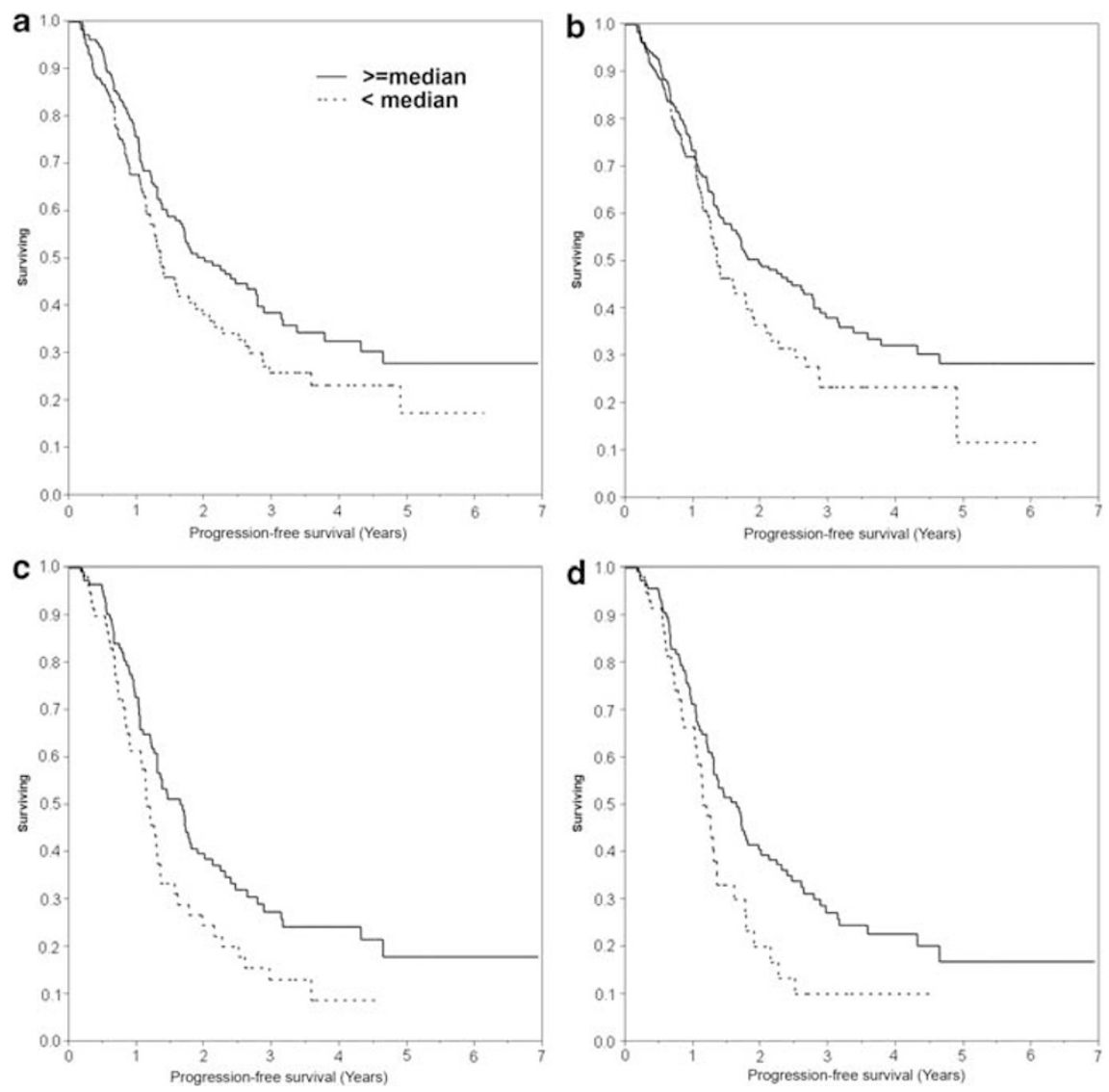

Figure 6 (a, b) Progression-free survival analysis of MCM7 score by the observer (a) and by the automated cellular imaging system (ACIS) (b) in the entire tumor cohort. (c, d) Progression-free survival analysis of MCM7 score by the observer (c) and by the ACIS (d) in patients with high-grade serous carcinomas. Solid lines represent MCM7 scores equal to or above the median, and dashed lines represent MCM7 scores below median. 
Table 5 Effect likelihood ratio tests for progression-free survival

\begin{tabular}{lccccccrrr}
\hline Source & Nparm & $D F$ & $L R \chi^{2}$ & Prob $>\chi^{2}$ & Source & Nparm & DF & $L R \chi^{2}$ & Prob $>\chi^{2}$ \\
\hline Stage & 3 & 3 & 43.00 & $<\mathbf{0 . 0 0 0 1}$ & Stage & 3 & 3 & 44.6 \\
Histology & 3 & 3 & 8.10 & $\mathbf{0 . 0 4 4}$ & Histology & 3 & 3 & 7.5 \\
MCM7 & 1 & 1 & 2.59 & 0.11 & MCM7 & 1 & $\mathbf{0 . 0 0 0 1}$ \\
\end{tabular}

ACIS, automated cellular imaging system; DF, degree of freedom; LR, likelihood ratio; Nparm, number of parameters; Observer, pathologist observer; Prob, probability.

Significant associations are highlighted by bold letters.

Table 6 Risk ratios for MCM7 labeling index ${ }^{\mathrm{a}}$

\begin{tabular}{lcccc}
\hline Term & Risk ratio & $\begin{array}{c}95 \% \\
\text { Lower } C L\end{array}$ & $\begin{array}{c}95 \% \\
\text { Upper } C L\end{array}$ & Reciprocal \\
\hline Observer & 0.63 & 0.35 & 1.1 & 1.6 \\
ACIS & 0.4 & 0.2 & 0.89 & 2.34 \\
\hline
\end{tabular}

ACIS, automated cellular imaging system; CL, confidence limit; MCM7, minichromosome maintenance protein 7; Observer, pathologist observer.

${ }^{a}$ Per change in regressor over entire range.

Table 7 Risk ratios by stage and histology

\begin{tabular}{|c|c|c|c|c|c|}
\hline \multicolumn{3}{|c|}{ Observer } & \multicolumn{3}{|c|}{ ACIS } \\
\hline Stage & /Stage & $\begin{array}{l}\text { Risk } \\
\text { ratio }\end{array}$ & Stage & /Stage & $\begin{array}{l}\text { Risk } \\
\text { ratio }\end{array}$ \\
\hline 2 & 1 & 1.23 & 2 & 1 & 1.23 \\
\hline 3 & 1 & 6.84 & 3 & 1 & 7.03 \\
\hline 3 & 2 & 5.56 & 3 & 2 & 5.72 \\
\hline 4 & 1 & 10.94 & 4 & 1 & 11.64 \\
\hline 4 & 2 & 8.89 & 4 & 2 & 9.47 \\
\hline 4 & 3 & 1.60 & 4 & 3 & 1.66 \\
\hline \multicolumn{3}{|c|}{ Observer } & \multicolumn{3}{|c|}{ ACIS } \\
\hline Histology & /Histology & $\begin{array}{l}\text { Risk } \\
\text { ratio }\end{array}$ & Histology & /Hisotology & $\begin{array}{l}\text { Risk } \\
\text { ratio }\end{array}$ \\
\hline 2 & 1 & 0.27 & 2 & 1 & 0.30 \\
\hline 3 & 1 & 1.64 & 3 & 1 & 1.69 \\
\hline 3 & 2 & 6.02 & 3 & 2 & 5.62 \\
\hline 4 & 1 & 1.25 & 4 & 1 & 1.29 \\
\hline 4 & 2 & 4.57 & 4 & 2 & 4.29 \\
\hline 4 & 3 & 0.76 & 4 & 3 & 0.76 \\
\hline
\end{tabular}

ACIS, automated cellular imaging system; Observer, pathologist observer.

Histology code: $1=$ serous, $2=$ endometrioid, $3=$ clear cell, and $4=$ mucinous .

index showed strong concordance between the observer-derived labeling index and the ACISderived labeling index. Analysis of MCM7 expression in different histological subtypes of ovarian tumors further confirmed our previous cDNA expression studies indicating higher levels of MCM7 expression in high-grade serous carcinomas compared with borderline tumors. These results are significant, considering that previous cDNA expression studies were performed using formalin-fixed paraffin-embedded tumor samples. ${ }^{8}$ In this study, we also observed significantly higher expression of MCM7 in high-grade serous carcinomas compared with endometrioid and clear-cell tumors. It should be noted that we also observed variable levels of MCM7 expression in other histo-subtypes, although the clinical significance of such variable expression in these histo-subtypes is unclear. Our observation that MCM7 expression in clear-cell carcinomas is lower than in high-grade serous tumors is important, considering that clear-cell carcinomas also showed increased risk of recurrence in our sample cohort. Given that recurrence is in part associated with poor response to chemotherapy, it is reasonable to suggest that low levels of MCM7 expression may be associated with low proliferative capacity and poor response to chemotherapy in clear-cell carcinomas. Therefore, these results provide possible functional links between lower MCM7 expression and poor response to chemotherapy and consequent increased risk of recurrence. Moreover, it is interesting to note that increased risk of recurrence is observed in patients with clear-cell tumors compared with patients with high-grade serous carcinomas, despite the fact that the majority of clear-cell tumors were diagnosed at early stages and the majority of highgrade serous carcinomas were diagnosed at advanced stages (Table 7). These results are consistent with several reports indicating the poor response of clearcell carcinomas to platinum-based chemotherapy. ${ }^{26}$

The potential clinical importance of MCM7 expression is not limited to clear cells. For the entire tumor cohort, reduced MCM7 expression was associated with increased risk of recurrence (Tables 5 and 6). In addition, MCM7 expression was an independent prognostic factor for progression-free survival. In multivariate analyses that included stage, histology, and MCM7 labeling index, MCM7 expression was significantly associated with progression-free survival. When limiting the analysis to high-grade serous carcinomas, MCM7 expression was still significantly associated with progressionfree survival. In addition, when the analysis was limited to late-stage, high-grade serous carcinomas, the MCM7 labeling index produced by ACIS was significantly associated with progression-free survival. These results suggest the prognostic value of the MCM7 labeling index in late-stage, high-grade serous carcinomas and the potential use of ACISbased MCM7 expression analyses in translational and clinical studies. 
In conclusion, our results demonstrate MCM7 expression as the potential prognostic biomarker for disease recurrence or progression-free survival in ovarian cancer. Additional studies are needed to establish the role of MCM7 in the differential response to chemotherapy. Nonetheless, analysis of progression-free survival in relation to MCM7 expression indicates MCM7 expression could be used as a prognostic factor in patients' response to chemotherapy.

\section{Acknowledgements}

We thank Steve Kalloger for his assistance with compiling clinical information and statistical support. This work was supported by a Simmers Career Development Award, grants from the Andersen Foundation and JW Anderson (to JRC) and the Ovarian Cancer Research Fund (to TO), and Grant 1 UL1 RR024150 from the National Center for Research Resources (NCRR), a component of the National Institutes of Health (NIH), and the NIH Roadmap for Medical Research.

\section{Disclaimer}

The contents of this study are solely the responsibility of the authors and do not necessarily represent the official view of NCRR or NIH.

\section{Disclosure/conflict of interest}

The authors declare no conflict of interest.

\section{References}

1 Jemal A, Siegel R, Ward E, et al. Cancer statistics, 2009. CA Cancer J Clin 2009;59:225-249.

2 Gadducci A, Fuso L, Cosio S, et al. Are surveillance procedures of clinical benefit for patients treated for ovarian cancer?: A retrospective Italian multicentric study. Int J Gynecol Cancer 2009;19:367-374.

3 Sabatier R, Finetti P, Cervera N, et al. Gene expression profiling and prediction of clinical outcome in ovarian cancer. Crit Rev Oncol Hematol 2009;72:98-109.

4 Gadducci A, Cosio S, Tana R, et al. Serum and tissue biomarkers as predictive and prognostic variables in epithelial ovarian cancer. Crit Rev Oncol Hematol 2009;69:12-27.

5 Gilks CB, Vanderhyden BC, Zhu S, et al. Distinction between serous tumors of low malignant potential and serous carcinomas based on global mRNA expression profiling. Gynecol Oncol 2005;96:684-694.

6 Bonome T, Lee JY, Park DC, et al. Expression profiling of serous low malignant potential, low-grade, and high-grade tumors of the ovary. Cancer Res 2005;65: 10602-10612.

7 Marchini S, Mariani P, Chiorino G, et al. Analysis of gene expression in early-stage ovarian cancer. Clin Cancer Res 2008;14:7850-7860.
8 Chien J, Fan JB, Bell DA, et al. Analysis of gene expression in stage I serous tumors identifies critical pathways altered in ovarian cancer. Gynecol Oncol 2009;114:3-11.

9 Tye BK. MCM proteins in DNA replication. Annu Rev Biochem 1999;68:649-686.

10 Forsburg SL. Eukaryotic MCM proteins: beyond replication initiation. Microbiol Mol Biol Rev 2004;68:109-131.

11 Forsburg SL. The MCM helicase: linking checkpoints to the replication fork. Biochem Soc Trans 2008;36: 114-119.

12 Li SS, Xue WC, Khoo US, et al. Replicative MCM7 protein as a proliferation marker in endometrial carcinoma: a tissue microarray and clinicopathological analysis. Histopathology 2005;46:307-313.

13 Ren B, Yu G, Tseng GC, et al. MCM7 amplification and overexpression are associated with prostate cancer progression. Oncogene 2006;25:1090-1098.

14 Tsai HY, Hsi BL, Hung IJ, et al. Correlation of MYCN amplification with MCM7 protein expression in neuroblastomas: a chromogenic in situ hybridization study in paraffin sections. Hum Pathol 2004;35:1397-1403.

15 Nishihara K, Shomori K, Fujioka S, et al. Minichromosome maintenance protein 7 in colorectal cancer: implication of prognostic significance. Int J Oncol 2008;33:245-251.

16 Fujioka S, Shomori K, Nishihara K, et al. Expression of minichromosome maintenance 7 (MCM7) in small lung adenocarcinomas (pT1): Prognostic implication. Lung Cancer 2009;65:223-229.

17 Facoetti A, Ranza E, Benericetti E, et al. Minichromosome maintenance protein 7: a reliable tool for glioblastoma proliferation index. Anticancer Res 2006; 26:1071-1075.

18 Gambichler T, Breininger A, Rotterdam S, et al. Expression of minichromosome maintenance proteins in Merkel cell carcinoma. J Eur Acad Dermatol Venereol 2009;23:1184-1188.

19 Goodman MT, Shvetsov YB. Incidence of ovarian, peritoneal, and fallopian tube carcinomas in the United States, 1995-2004. Cancer Epidemiol Biomarkers Prev 2009;18:132-139.

20 Suzuki S, Adachi A, Hiraiwa A, et al. Cloning and characterization of human MCM7 promoter. Gene 1998;216:85-91.

21 Ohtani K, Iwanaga R, Nakamura M, et al. Cell growthregulated expression of mammalian MCM5 and MCM6 genes mediated by the transcription factor E2F. Oncogene 1999;18:2299-2309.

22 Tye BK, Chang VK. Dual functional regulators coordinate DNA replication and gene expression in proliferating cells. Front Biosci 2004;9:2548-2555.

23 Shohet JM, Hicks MJ, Plon SE, et al. Minichromosome maintenance protein MCM7 is a direct target of the MYCN transcription factor in neuroblastoma. Cancer Res 2002;62:1123-1128.

24 Stoeber K, Tlsty TD, Happerfield L, et al. DNA replication licensing and human cell proliferation. J Cell Sci 2001;114:2027-2041.

25 Mayer A, Takimoto M, Fritz E, et al. The prognostic significance of proliferating cell nuclear antigen, epidermal growth factor receptor, and mdr gene expression in colorectal cancer. Cancer 1993;71:2454-2460.

26 Tan DS, Kaye S. Ovarian clear cell adenocarcinoma: a continuing enigma. J Clin Pathol 2007;60:355-360. 